\title{
Establishing a multidisciplinary AIDS-associated Kaposi's sarcoma clinic: Patient characteristics, management and outcomes
}

\author{
H Burger, ${ }^{1}$ MB ChB, PG Dip Pall Med (SA), FC Rad Onc (SA); Z Ismail, ${ }^{2}$ MB ChB, PG Dip HIV Man (SA); \\ J J Taljaard, ${ }^{2} \mathrm{MB} \mathrm{ChB}$, MMed (Internal Medicine), DTM\&H
${ }^{1}$ Division of Radiation Oncology, Department of Medical Imaging and Clinical Oncology, Tygerberg Academic Hospital and Faculty of Medicine and Health Sciences, Stellenbosch University, Cape Town, South Africa
${ }^{2}$ Division of Infectious Diseases, Department of Internal Medicine, Tygerberg Academic Hospital and Faculty of Medicine and Health Sciences, \\ Stellenbosch University, Cape Town, South Africa
}

Corresponding author: H Burger (henrietteburger@sun.ac.za)

\begin{abstract}
Background. Kaposi's sarcoma (KS) typically occurs in the setting of immunodeficiency and specifically in the presence of HIV infection, when it is called AIDS-associated KS (AIDS-KS). In spite of impressive gains in the South African (SA) antiretroviral therapy (ART) roll-out programme since 2004, AIDS-KS still causes significant morbidity and mortality, and the treatment of advanced disease can be challenging owing to the centralisation of oncology services and the high incidence of concurrent infections. In 2014, a multidisciplinary AIDS-KS clinic (MKSC) was established at Tygerberg Hospital, Cape Town, with the goal of optimising management of AIDS-KS patients.

Objectives. To report on the characteristics and outcomes of patients seen during the first 6 months after the inception of the MKSC. Methods. A retrospective observational study was performed of all new cases referred to the MKSC from February to August 2014. Results. Forty-two patients were included in the study. The median age was 34 years (range 20 - 60). Forty-one patients were on ART at time of diagnosis or were initiated by a median of 3 months after diagnosis. The median CD4+ count before diagnosis was 147 cells/ $\mu \mathrm{L}$ (range 4 811). The HIV viral load was undetectable in 22 cases (52.4\%). Thirty-eight patients (90.5\%) were classified as AIDS Clinical Trials Group (ACTG) poor risk, 10 patients $(23.8 \%)$ had visceral KS, 14 patients (33.3\%) were on tuberculosis (TB) treatment at time of presentation, and 22 patients $(52.4 \%)$ received oncological therapy in addition to ART. After median follow-up of 25.6 months, 2-year overall survival (OS) was $61.1 \%$. On univariate analysis, factors significantly associated with poor 2-year OS included ACTG S1 stage ( $\mathrm{S}=$ systemic illness), visceral KS, being on TB treatment, and Eastern Cooperative Oncology Group performance status score $>2$. In the T1 $(\mathrm{T}=$ tumour extent) subgroup, receiving chemotherapy was significantly associated with improved 2-year OS.

Conclusions. Advanced AIDS-KS significantly affects young people in the Western Cape Province of SA despite 10 years of ART roll-out. There is a high prevalence of concomitant TB infection that could adversely affect adherence and response to treatment. Despite advanced disease at presentation and palliative treatment intent, survival outcomes are encouraging and seem to be positively affected by the increased use of chemotherapy. A multidisciplinary approach to diagnosis, staging and treatment and the exploration of prognostic indices specific to the sub-Saharan setting would be valuable in designing appropriate treatment algorithms.
\end{abstract}

S Afr Med J 2018;108(12):1059-1065. DOI:10.7196/SAMJ.2018.v108i12.13202

Kaposi's sarcoma (KS) is a multicentric angioproliferative malignancy of endothelial cell origin associated with human herpesvirus 8 infection. KS typically occurs in the setting of immunodeficiency and specifically in the presence of HIV infection, when it is called AIDS-associated KS (AIDS-KS). AIDS-KS is classified as an AIDS-defining cancer. It is characterised by a wide variation in clinical presentations and course of disease. Since the widespread introduction of antiretroviral therapy (ART) in Europe and North America in the 1990s, the incidence of and mortality from AIDS-KS have decreased dramatically. ${ }^{[1]}$ Similar trends have been reported in sub-Saharan countries, but in spite of impressive gains in the South African (SA) ART roll-out programme since 2004, AIDS-KS remains a condition that causes significant morbidity and mortality in the SA HIV-infected population. ${ }^{[2-7]}$ A recent publication estimated the SA AIDS-KS incidence rate in patients recently initiated on ART as 138 per 100000 person-years. ${ }^{[8]}$ The modified AIDS Clinical Trials Group (ACTG) staging system classifies AIDS-KS as either limited disease with a good prognosis or advanced disease with a poorer prognosis. ${ }^{[9]}$
The treatment of advanced AIDS-KS can be challenging in SA owing to the centralisation of oncology services to tertiary hospitals and the high incidence of concurrent opportunistic infections (OIs). ${ }^{[10]}$ The Tygerberg Hospital (TBH) infectious diseases clinic is a tertiary referral centre in a high HIV prevalence setting in Cape Town, SA. In 2014, a weekly multidisciplinary AIDS-KS clinic (MKSC) was established in collaboration with the TBH oncology division to evaluate all new cases of AIDS-KS with the goal of optimising management of patients in the drainage area by improving access to these specialist clinical services. A management and referral standard operating procedure was developed and the MKSC was promoted to referring clinics via provincial structures.

\section{Objectives}

To describe patients with AIDS-KS referred to the MKSC in terms of demographic and disease characteristics, treatment and outcomes, and to analyse the associations between these factors. The primary objective was to report on overall survival (OS) from diagnosis with 
KS for the entire cohort. The secondary objectives were to report on the 2-year OS from diagnosis with KS for selected subgroups and on statistical associations between selected clinically relevant demographic, disease or treatment characteristics and the primary outcome.

\section{Methods}

The clinical records of all patients with AIDS-KS assessed at the MKSC between February and August 2014 were retrospectively reviewed. Patients were excluded if they had a second malignancy that required more urgent therapy than their KS. Data collected included patient demographics (age at diagnosis, gender and clinician-reported ethnic group), ART initiation and duration, CD4+ count at diagnosis, HIV viral load (VL) at referral, presence of OIs, histological findings, KS stage according to the modified ACTG staging system (Table 1) and sites of involvement, presence of oedema, performance status (PS), and treatment modalities and responses. ${ }^{[9]}$ Survival outcomes were defined as death, loss to follow-up (LTFU) or alive, and were censored on 4 September 2016. LTFU status was assigned if the living status of a patient could not be determined on the censoring date. These patients were censored on the date of last attendance. Time to death or LTFU was defined as number of months from diagnosis of KS. Living status was determined from hospital records and the national death registry. Descriptive statistics were used to report the prevalence of individual variables and the Kaplan-Meier method was used to estimate survival outcomes. The log-rank test was used to assess differences in survival distributions. Incidence rate ratios (IRRs) and $95 \%$ confidence intervals (CIs) were calculated. A p-value of $<0.05$ was considered statistically significant. Ethics approval was obtained from the Stellenbosch University Human Research Ethics committee (ref. no. M15/10/048).

\section{Results}

A total of 42 patients with a median age at AIDS-KS diagnosis of 34 years (range $20-60$ ) were included in the analysis. Twenty-three patients $(54.8 \%)$ were female. Thirty-six patients $(85.7 \%)$ were black, and the remainder were coloured.

Twenty-eight patients (66.7\%) were on ART before KS diagnosis. The median number of weeks on ART was 12.5 (range 0 - 643). A further 13 patients (30.9\%) were started on ART within a median of 3 weeks after KS diagnosis, bringing the total number of patients on ART to 41 (97.6\%). The specific ART regimens were not recorded. The median CD4+ count at the time of diagnosis was 147 cells/ $\mu \mathrm{L}$ (range $4-811$ ), with 21 patients $(50 \%)$ having a CD4+ count $<150$ cells $/ \mu \mathrm{L}$. VL at time of referral to the MKSC was known to be undetectable in 22 cases (52.4\%) and was unknown in 12 cases (28.6\%).

Forty patients (95.2\%) had histologically confirmed KS. The remaining 2 patients were diagnosed clinically. Four patients (9.5\%) were classified as ACTG good risk (TOI0S0, where $\mathrm{T}=$ tumour extent, $\mathrm{I}=$ immune status and $\mathrm{S}=$ systemic illness) and $38(90.5 \%)$ as poor risk. Thirty-six patients (85.7\%) were T1 and 20 (47.6\%) were S1. The S1 cohort represents the T1S1 cohort, as there were no cases of T0S1 disease. Seven patients (16.7\%) had an Eastern Cooperative Oncology Group (ECOG) PS score of $>2$. ECOG PS was undocumented in 17 cases $(40.5 \%)$. The most common sites of involvement were the skin of the lower limbs ( $n=26$ cases, $61.9 \%$ ) and the oral mucosa $(n=21,50.0 \%)$. Twenty-one patients $(50.0 \%)$ had documented oedema. Ten patients $(23.8 \%)$ had visceral KS, 3 with pulmonary KS (radiological diagnosis), 6 with gastrointestinal tract (GIT) KS (endoscopic diagnosis) and 1 with both.

Fourteen patients $(33.3 \%)$ were on TB treatment at time of referral to the MKSC. Other co-infections recorded included syphilis, toxoplasmosis, cryptococcal meningitis, oral candida, Klebsiella pneumonia and superficial infection of KS skin lesions.

All treatment decisions were made by the multidisciplinary team (MDT) consisting of infectious diseases physicians, clinical oncologists and dermatologists. Twenty patients (47.6\%) received ART only and $22(52.4 \%)$ received ART and oncological therapy (Table 2).

Three distinct treatment groups were identified retrospectively based on the type of therapy received as recommended by the MDT (Table 3$)$. Ten patients had T0 $(n=6)$ or T1 $(n=4)$ disease that was responding clinically to ART and received no oncological treatment in addition to continued ART (group 1). This group included 2 patients with T1S1 disease. The remaining 32 patients had symptomatic T1 ( $n=18 \mathrm{~T} 1 \mathrm{~S} 1$ ) disease that was not responding to ART adequately and were considered for oncotherapy. Of these patients, 22 ( $n=9$ T1S1) received additional oncological treatment (group 2). Six were on TB treatment and 6 had visceral KS. Thirteen patients had chemotherapy alone, 3 had radiotherapy (RT) alone and 6 had both. The median dose of RT was 8 Gy (range 6 - 30.25). Three patients had multiple sites of RT. One patient underwent intraocular bleomycin administration and the remainder received systemic combination chemotherapy. Two patients received second-line chemotherapy for progressive AIDS-KS. The remaining 10 patients $(n=9 \mathrm{~T} 1 \mathrm{~S} 1)$ did not receive oncological treatment in addition to ART because they either defaulted from care $(n=2)$ or were found to be unfit for oncological therapy ( $n=8$ ) (group 3). Six of these patients were on TB treatment and 4 had visceral KS.

\section{Table 1. ACTG modified staging classification for AIDS-KS ${ }^{[9]}$}

\begin{tabular}{|c|c|c|}
\hline & Good risk (all of the following) & Poor risk (any of the following) \\
\hline $\mathrm{T}$ & $\begin{array}{l}\text { T0: confined to skin and/or lymph nodes and/or minimal } \\
\text { oral disease (non-nodular KS confined to palate) }\end{array}$ & $\begin{array}{l}\text { T1: tumour-associated oedema or ulceration } \\
\text { Extensive oral KS } \\
\text { Gastrointestinal KS } \\
\text { KS in other non-nodal viscera }\end{array}$ \\
\hline I & I0: CD4+ cell count $>150 / \mu \mathrm{L}$ & I1: CD $4+$ cell count $<150 / \mu \mathrm{L}$ \\
\hline S & $\begin{array}{l}\text { S0: no history of OI or thrush } \\
\text { No 'B' symptoms } \\
\text { Karnofsky PS }>70\end{array}$ & $\begin{array}{l}\text { S1: history of OI and/or thrush } \\
\text { 'B' symptoms present } \\
\text { Karnofsky PS }<70 \\
\text { Other HIV-related illness (e.g. neurological disease, } \\
\text { lymphoma) }\end{array}$ \\
\hline
\end{tabular}




\begin{tabular}{|c|c|}
\hline Age (years), median (range) & $34(20-60)$ \\
\hline \multicolumn{2}{|l|}{ Gender, $n(\%)$} \\
\hline Female & $23(54.8)$ \\
\hline Male & $19(45.2)$ \\
\hline \multicolumn{2}{|l|}{ Ethnic group, $n(\%)$} \\
\hline Black & $36(85.7)$ \\
\hline Coloured & $6(14.3)$ \\
\hline \multicolumn{2}{|l|}{ ART initiation, $n$ (\%) } \\
\hline Before KS diagnosis & $28(66.7)$ \\
\hline After KS diagnosis & $13(30.9)$ \\
\hline Not initiated & $1(2.4)$ \\
\hline \multicolumn{2}{|l|}{ CD $4+$ count $($ cells $/ \mu \mathrm{L})$} \\
\hline Median (range) & $147(4-811)$ \\
\hline$<150, n(\%)$ & $21(50.0)$ \\
\hline \multicolumn{2}{|l|}{ VL undetectable, $n(\%)$} \\
\hline Yes & $22(52.4)$ \\
\hline No & $8(19.0)$ \\
\hline Unknown & $12(28.6)$ \\
\hline \multicolumn{2}{|l|}{ KS stage, $n(\%)$} \\
\hline Good risk & $4(9.5)$ \\
\hline Poor risk & $38(90.5)$ \\
\hline T0 & $6(14.3)$ \\
\hline $\mathrm{T} 1$ & $36(85.7)$ \\
\hline So & $22(52.4)$ \\
\hline $\mathrm{S} 1(\mathrm{~T} 1 \mathrm{~S} 1)^{*}$ & $20(47.6)$ \\
\hline \multicolumn{2}{|l|}{ ECOG PS, $n(\%)$} \\
\hline$\leq 2$ & $18(42.9)$ \\
\hline$>2$ & $7(16.7)$ \\
\hline Unknown & $17(40.5)$ \\
\hline \multicolumn{2}{|l|}{ Site of KS involvement, $n$ (\%) } \\
\hline Lower limb & $26(61.9)$ \\
\hline Oral cavity & $21(50.0)$ \\
\hline Oedema & $21(50.0)$ \\
\hline \multicolumn{2}{|l|}{ Visceral KS, $n(\%)$} \\
\hline Any & $10(23.8)$ \\
\hline $\mathrm{GIT}^{\dagger}$ & $7(16.7)$ \\
\hline Pulmonary & $4(11.9)$ \\
\hline \multicolumn{2}{|l|}{ OI, $n(\%)$} \\
\hline $\mathrm{TB}^{s}$ & $14(33.3)$ \\
\hline Other OI & $4(9.5)$ \\
\hline \multicolumn{2}{|l|}{ Treatment, $n(\%)$} \\
\hline ART & $41(97.6)$ \\
\hline Chemotherapy & $19(45.2)$ \\
\hline Radiotherapy & $9(21.4)$ \\
\hline \multicolumn{2}{|c|}{ 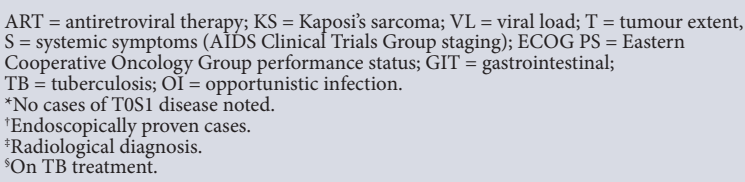 } \\
\hline
\end{tabular}

Response to RT or chemotherapy was assessed at 6 weeks from completion of treatment. Patients with lasting complete or partial response (PR) from baseline were referred to their ART clinic for follow-up with instructions to refer the patient back if progressive disease was noted. The attendance rate at 6 weeks after RT was 44.4\%. All these patients had PR as defined by any improvement
Table 3. Retrospective treatment groups according to $\mathrm{T}$ stage

\begin{tabular}{lll}
\hline Treatment group & Treatment & ACTG T stage, $\boldsymbol{n}$ \\
\hline 1 & ART alone & T0 6, T1 4 \\
2 & ART and RT and/or & T0 0, T1 22 \\
& chemotherapy & \\
3 & ART alone & T0 0, T1 10 \\
$\begin{array}{l}\mathrm{T}=\text { tumour extent; ACTG = AIDS Clinical Trials Group; ART = antiretroviral therapy; } \\
\text { RT = radiotherapy. }\end{array}$ &
\end{tabular}

Table 4. OS according to demographic and clinical factors

\begin{tabular}{|c|c|c|}
\hline & $\begin{array}{l}\text { 2-year } \\
\text { OS from } \\
\text { diagnosis, \% }\end{array}$ & $p$-value (IRR, 95\% CI) \\
\hline All $(N=42)$ & 61.1 & \\
\hline ACTG S1 (T1S1) & & $0.001(5.88,1.78-25.0)$ \\
\hline Yes $(n=20)$ & 38.5 & \\
\hline No $(n=22)$ & 81.3 & \\
\hline Known visceral KS & & $0.03(3.53,1.05-10.71)$ \\
\hline Yes $(n=10)$ & 36.0 & \\
\hline No $(n=32)$ & 68.4 & \\
\hline Known pulmonary KS & & $0.004(11.54,2.11-42.01)$ \\
\hline Yes $(n=4)$ & 0 & \\
\hline No $(n=38)$ & 64.9 & \\
\hline Known ECOG PS $>2$ & & $0.01(6.2,1.34-31.4)$ \\
\hline Yes $(n=7)$ & 28.6 & \\
\hline No $(n=35)$ & 67.7 & \\
\hline Any OI & & $0.0005(6.29,2.01-23.08)$ \\
\hline Yes $(n=17)$ & 33.1 & \\
\hline No $(n=25)$ & 79.6 & \\
\hline On TB treatment & & $0.0031(4.67,1.55-14.74)$ \\
\hline Yes $(n=14)$ & 32.7 & \\
\hline No $(n=28)$ & 74.7 & \\
\hline $\begin{array}{l}\text { OS = overall survival; } \mathrm{IRR}=\text { inc } \\
\text { Clinical Trials Group; } \mathrm{T}=\text { tumo } \\
\text { ECOG PS = Eastern Cooperativ } \\
\text { infection; } \mathrm{TB}=\text { tuberculosis. }\end{array}$ & $\begin{array}{l}\text { cidence rate ratio; } \mathrm{Cl} \\
\text { uru extent; } \mathrm{S}=\text { systen } \\
\text { ve Oncology Group }\end{array}$ & $\begin{array}{l}=\text { confidence interval; } \mathrm{ACTG}=\mathrm{AIDS} \\
\text { hic symptoms; } \mathrm{KS}=\mathrm{Kaposi} \text { s sarcoma } \\
\text { performance status; } \mathrm{OI}=\text { opportunisti }\end{array}$ \\
\hline
\end{tabular}

in symptoms or reduction in size of target lesions lasting for 6 weeks after completion of treatment. All but one of the patients treated with systemic therapy received adriamycin $\left(40 \mathrm{mg} / \mathrm{mm}^{2}\right)$, bleomycin $\left(10 \mathrm{IU} / \mathrm{mm}^{2}\right)$ and vinblastine $\left(6 \mathrm{mg} / \mathrm{mm}^{2}\right)$ combination chemotherapy prescribed on a 4 -weekly basis for 6 cycles. One patient received bleomycin $\left(15 \mathrm{IU} / \mathrm{mm}^{2}\right)$ and vincristine $(2 \mathrm{mg})$ given on a 3-weekly basis owing to a change in departmental protocol from 2015. Nine of 19 patients (47.4\%) completed the intended number of cycles (median 4, range $1-8$ ). A significant delay of 14 days or more was seen in only 4 patients (19.1\%) (median 3 days, range 0 37). Seven of the $19(36.8 \%)$ attended the follow-up visit 6 weeks after completing chemotherapy. Six (31.6\%) demonstrated PR and 1 (5.3\%) had stable disease. Overall, 7 patients (31.8\%) defaulted from oncological treatment, but 17 (77.3\%) defaulted from follow-up at some point. There were 2 deaths on chemotherapy. The exact cause of death was not known in either case.

After a median follow-up from diagnosis of 25.6 months (range 0.3 - 32.6), there had been 16 deaths (38.1\%). Cumulative 2-year OS from diagnosis was $61.1 \%$ and the median OS was not reached (Table 4). Cumulative 2-year OS for S0 and S1 disease was $81.3 \%$ and $38.5 \%$, respectively ( $p=0.001$, IRR $5.88,95 \%$ CI $1.78-25.0$ ) (Fig. 1). Patients without visceral disease had a cumulative 2-year 
OS of $68.4 \%$ compared with $36.0 \%$ in patients with visceral disease ( $p=0.03$, IRR 3.53, 95\% CI 1.05 - 10.71) (Fig. 2). Other demographic and disease factors that negatively affected survival outcome were

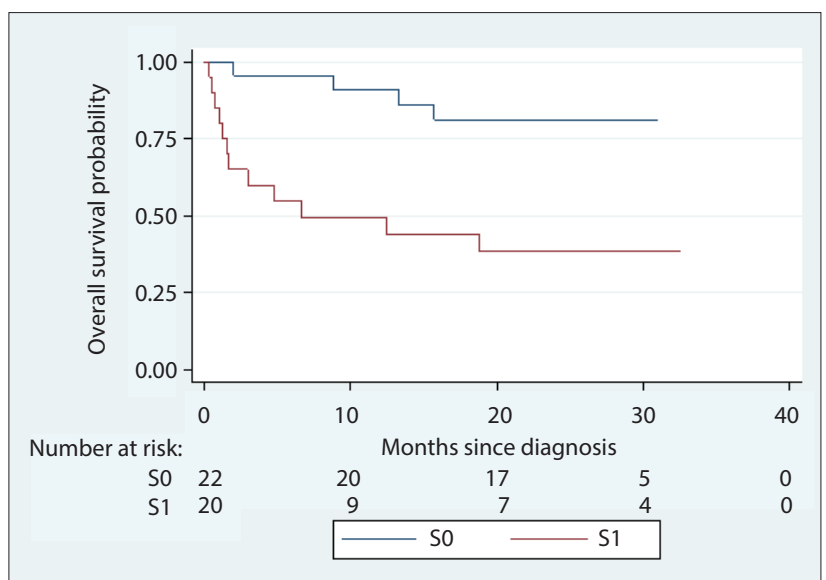

Fig. 1. Two-year overall survival according to $S$ stage $(\mathrm{p}<0.001)$. $(S=$ systemic illness.)

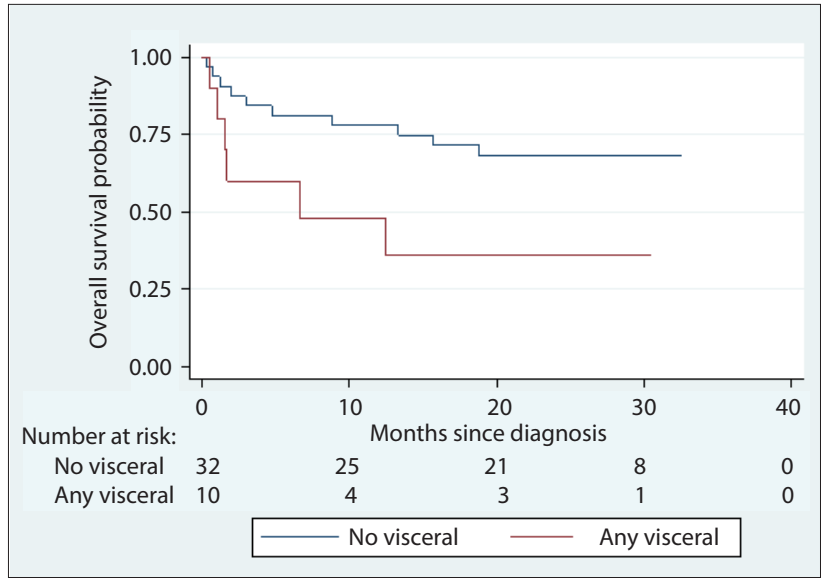

Fig. 2. Two-year overall survival according to visceral $K S(\mathrm{p}=0.03)$. $(K S=$ Kaposi's sarcoma.) pulmonary involvement, being on TB treatment, ECOG PS $>2$ and the presence of any OI (Table 4). T1 disease, I1 disease, ACTG poorrisk group, gender, ethnic group, ART before KS diagnosis, VL lower than detectable limit, oedema, specific skin sites, oral involvement and GIT involvement were not significantly associated with 2-year OS.

The 2-year OS according to treatment group was $90 \%, 70.9 \%$ and $10 \%$ for groups 1, 2 and 3, respectively $(p<0.001)$ (Table 5$)$. In the T1 subgroup, 2-year OS by treatment group was $100 \%, 70.9 \%$ and $10 \%$, respectively $(p<0.001)$ (Fig. 3 ). T1 patients receiving chemotherapy had a 2 -year OS of $77.3 \%$ v. $35.3 \%$ for those without chemotherapy $(p=0.0019$, IRR $0.18,95 \%$ CI $0.04-0.61)$. In the T1 subgroup not responding optimally to ART, the 2 -year OS was $15.4 \%(p<0.001$, IRR 0.09, 95\% CI 0.02 - 0.3) (Fig. 4).

\section{Discussion}

One of the challenges in treating advanced AIDS-KS is the lack of multidisciplinary input in the accurate diagnosis, staging and treatment selection of patients. ${ }^{[5]}$ During the first 6 months after its establishment, the MKSC assessed 42 new AIDS-KS patients.

The median age at KS diagnosis of 34 years is similar to that reported in three other SA cohorts. ${ }^{[5,11,12]}$ Although this finding can be expected

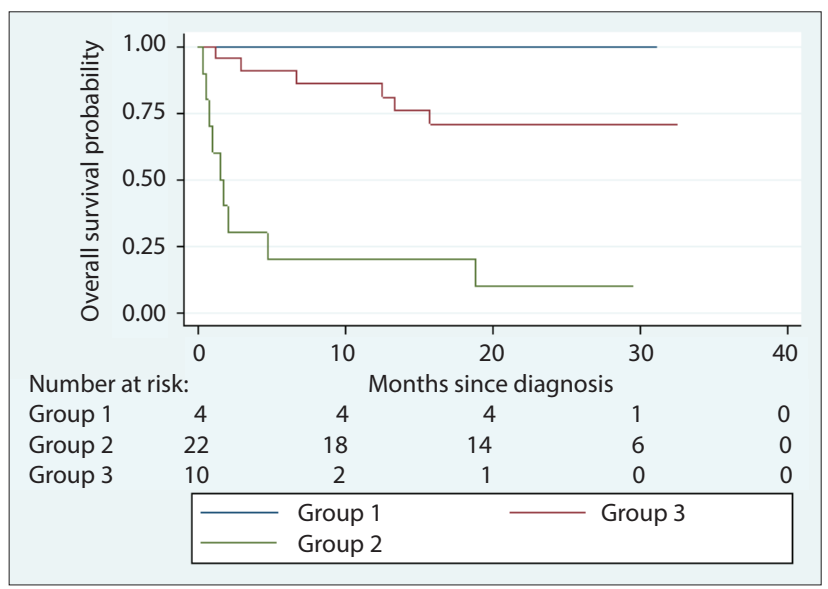

Fig. 3. Two-year overall survival in T1 according to treatment group (see text for description of the treatment groups) $(\mathrm{p}<0.001)$. ( $T=$ tumour extent. $)$

\section{Table 5. OS according to treatment}

\begin{tabular}{|c|c|c|c|c|c|c|}
\hline \multirow[b]{2}{*}{ Treatment type } & \multicolumn{2}{|c|}{ All $(N=42)$} & \multicolumn{2}{|r|}{$\mathrm{T} 1(N=36)$} & \multicolumn{2}{|c|}{$\begin{array}{l}\text { T1 with suboptimal response to ART } \\
\qquad(N=32)\end{array}$} \\
\hline & $\begin{array}{l}\text { 2-year } \\
\text { OS from } \\
\text { diagnosis (\%) }\end{array}$ & $\begin{array}{l}p \text {-value } \\
\text { (IRR, 95\% CI) }\end{array}$ & $\begin{array}{l}\text { 2-year } \\
\text { OS from } \\
\text { diagnosis (\%) }\end{array}$ & $\begin{array}{l}p \text {-value } \\
\text { (IRR, 95\% CI) }\end{array}$ & $\begin{array}{l}\text { 2-year } \\
\text { OS from } \\
\text { diagnosis (\%) }\end{array}$ & $\begin{array}{l}p \text {-value } \\
(\text { IRR, 95\% CI })\end{array}$ \\
\hline Treatment group ${ }^{*}$ & & $<0.001$ & & $<0.001$ & & $<0.001$ \\
\hline 1 & 90.0 & & 100 & & NA & \\
\hline 2 & 70.9 & & 70.9 & & 70.9 & \\
\hline 3 & 10.0 & & 10 & & 10.0 & \\
\hline Chemotherapy & & $\begin{array}{l}0.023 \\
(0.29,0.067-0.941)\end{array}$ & & $\begin{array}{l}0.0019 \\
(0.18,0.04-0.61)\end{array}$ & & $\begin{array}{l}<0.001 \\
(0.09,0.02-0.3)\end{array}$ \\
\hline Yes & 77.3 & & 77.3 & & 77.3 & \\
\hline No & 47.8 & & 35.3 & & 15.4 & \\
\hline Radiotherapy & & $\begin{array}{l}0.64 \\
(0.72,0.13-2.61)\end{array}$ & & $\begin{array}{l}0.41 \\
(0.58,0.1-2.14)\end{array}$ & & $\begin{array}{l}0.2 \\
(0.44,0.08-1.63)\end{array}$ \\
\hline Yes & 66.7 & & 66.7 & & 66.7 & \\
\hline No & 59.5 & & 54.0 & & 42.9 & \\
\hline
\end{tabular}




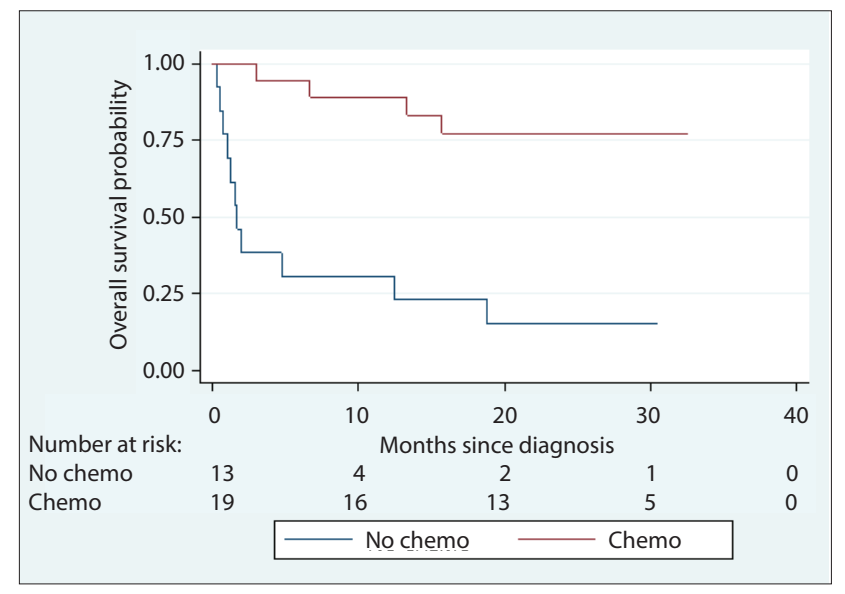

Fig. 4. Two-year overall survival according to chemotherapy in T1 with suboptimal response to $A R T(\mathrm{p}<0.001)$. (Chemo = chemotherapy; $T=$ tumour extent; $A R T=$ antiretroviral therapy.)

when considering the populations most burdened by HIV infection in SA, it highlights the burden of this advanced cancer in young people. The predominantly black racial distribution (85.7\%) is not in keeping with the demographics of the Western Cape, where $48.8 \%$ of the population is coloured and $32.8 \%$ is black. ${ }^{[13]}$ This points to the disproportionately high burden of AIDS-KS in black communities.

The median CD4+ count of 147 cells $/ \mu \mathrm{L}$ was low despite twothirds of the patients being on ART at time of diagnosis for a median of just over 3 months. All but one of the remaining patients who were not on ART at diagnosis commenced ART soon thereafter. This attests to effective local ART initiation policies and stands in contrast to an AIDS-KS cohort from the same geographical area in the pre- and early-ART era where only $47.3 \%$ of patients were on ART and the median CD4+ count was reported as 82 cells $/ \mu \mathrm{L} .{ }^{[5]}$ It is notable that although just over half of the patients had undetectable VLs at time of assessment by the MKSC, more than two-thirds still had suboptimal KS responses on ART alone. The wide variation in recorded $\mathrm{CD} 4+$ and VL values as well as strict policies on the timing of these tests make it very difficult to relate specific values and even trends to AIDS-KS prognosis in daily practice and may support a more complex immunological interaction, as suggested by Maurer (Unemori et al. ${ }^{[14]}$ ).

Fourteen patients (33.3\%) were on TB treatment and 4 had other OIs. It is important to note that some of these patients were empirically started on TB treatment despite negative sputum polymerase chain reaction (PCR) results, which could explain the higher rate of possible TB co-infection compared with the 19.1\% reported in a pre-ART cohort from KwaZulu-Natal Province ${ }^{[11]}$ but also calls into question the diagnosis of pulmonary TB (PTB) in patients who could have undiagnosed pulmonary KS. This scenario highlights the significant burden of disease and treatment toxicity carried by patients, who are often diagnosed with HIV, PTB and $\mathrm{KS}$ at the same time. The MDT approach proved crucial in making the difficult management decisions on the need for TB treatment in PCR-negative patients who may have pulmonary KS and require chemotherapy.

The large proportion of histological diagnoses (95.2\%) differs dramatically from the $3 \%$ reported for the same area a decade earlier. ${ }^{[5]}$ When the MKSC was started, all patients with AIDS-KS were seen regardless of $\mathrm{T}$ stage, but referral criteria have since been limited to T1 patients. Twenty patients (47.6\%) were T1S1, which has been reported to be a poor prognostic subgroup. ${ }^{[15]}$ High rates of lower limb and oral cavity involvement are in keeping with other SA cohorts. ${ }^{[5,6,11]}$ The relatively low number of recorded pulmonary KS cases $(11.9 \%)$ could be explained by the limited access to computed tomography scanning and the difficulty in distinguishing KS from PTB on plain chest films. Three of the 4 patients diagnosed with pulmonary KS were also on TB treatment. Pleuropulmonary KS is known to be a poor prognostic factor, and delayed diagnosis and treatment could have a negative impact on survival. ${ }^{[16]}$ The diagnosis of GIT involvement is made on endoscopy with or without biopsy. The MKSC referred all patients with lower than normal haemoglobin levels or suspicious GIT complaints for endoscopy to rule out gastrointestinal involvement. Data on KS-associated immune reconstitution inflammatory syndrome (KS-IRIS) were not recorded.

Despite lack of evidence of a survival benefit beyond that achieved by ART, guidelines recommend the early initiation of chemotherapy for advanced, symptomatic or rapidly progressive AIDS-KS as an effective means to palliate symptoms. ${ }^{[17]}$ The MKSC is regularly confronted with difficult treatment decisions that are complicated by late referrals, poor PS, serious co-infections and poor adherence to treatment. Twenty patients (47.6\%) did not receive oncotherapy. This group can be divided into two very different prognostic subgroups, namely patients with $\mathrm{T} 0 / \mathrm{T} 1$ disease responding clinically to ART, for which current guidelines suggest no additional therapy (group 1), ${ }^{[18]}$ and patients with $\mathrm{T} 1$ disease not responding to ART, who had clinical indications for oncotherapy but were either unfit for or defaulted from oncotherapy (group 3).

Oncotherapy was initiated in 22 patients (52.4\%) with T1 disease who were not responding optimally to ART alone (group 2). Primary treatment was chemotherapy in 14 cases (33.3\%). Despite less than half the patients completing the intended course, the response at 6 weeks for those who did attend scheduled follow-up was good. Primary therapy was RT in 8 cases $(19.0 \%)$ and 5 patients went on to receive chemotherapy after RT, starting within 6 weeks of $\mathrm{RT}$ in 4 cases. In these cases, RT was intended to gain rapid local symptom control in advanced lesions and follow-on chemotherapy was planned to control extensive disease. Despite poor attendance at 6 weeks after RT (44.4\%), all patients had a PR. One patient received concurrent chemoradiation to $30.25 \mathrm{~Gy}$.

The proportion of $\mathrm{T} 1$ patients receiving chemotherapy (52.8\%) and RT (25.0\%) differs from treatment patterns previously reported in the same geographical area and in Gauteng Province, in that patients were more likely to receive chemotherapy than $\mathrm{RT} \cdot{ }^{[5,6]}$ This is in keeping with international guidelines and the current protocol of the MKSC in which chemotherapy with combination bleomycin and vincristine or paclitaxel as single agent is used in all patients considered fit for chemotherapy. Radiotherapy is reserved for chemoresistant or highly localised lesions, or patients with lifethreatening localised lesions when chemotherapy cannot be started urgently ${ }^{[17]}$ High rates of defaulting from oncology treatment (31.8\%) and scheduled follow-up (77.3\%) pose a significant challenge in the treatment of AIDS-KS in our cohort.

Our cumulative 2-year OS of $61.1 \%$ compares favourably with most other SA cohorts in the post-ART era and may suggest improvement in local outcomes. ${ }^{[5,12,19]}$ Recently a 2 -year OS rate of $79 \%$ was reported in an SA cohort treated in the late ART era, but this cohort included only $56.6 \%$ poor-risk patients compared with the $90.5 \%$ reported here and excluded patients with visceral disease. ${ }^{[6]}$ A Nigerian AIDS-KS cohort with minimal access to chemotherapy reported a 1 -year mortality rate of $22 \%$ and 1 -year OS of $50-55 \%{ }^{[20]}$ Overall, the outcomes are still markedly inferior to those of international cohorts. ${ }^{[15,21]}$ When stratifying 2 -year OS by 
the predefined treatment groups, a statistically significant difference is seen favouring groups 1 and $2(p=0.0001)$ that persists when the T1 subgroup is analysed. The 2-year OS for patients with a suboptimal response to ART and receiving oncotherapy (group 2) was $70.9 \%$, which is remarkable considering that $40.0 \%$ were ACTG T1S1 and nearly a third had visceral disease or were on TB treatment. Patients with a suboptimal response to ART but not receiving oncotherapy (group 3) had a 2-year OS of $10 \%$ with only $30.0 \%$ surviving beyond 3 months. This can probably be ascribed to delays in presentation and referral, but may also signify cases of KS-IRIS.

Despite retrospective cohorts suggesting that the addition of chemotherapy to ART may improve survival, this has not been prospectively validated. ${ }^{[5,6,19]}$ In the $\mathrm{T} 1$ cohort, the addition of chemotherapy to ART improved 2-year OS markedly from $35.3 \%$ to $77.3 \%$. When T1 patients who were responding optimally to ART are excluded, 2-year OS without chemotherapy falls to $15.4 \%$. This suggests that the timely addition of chemotherapy to ART could confer a survival advantage in T1 AIDS-KS. This finding should be interpreted with caution, as bias due to early deaths and lack of ART adherence data in group 3 could exist. There was a nonsignificant trend towards a 2 -year OS benefit for chemotherapy in the T1S1 subgroup ( $62.5 \%$ v. $28.6 \%)$, a group that is reported to have a particularly poor prognosis. ${ }^{[15]}$ On a practical level, this addresses the often difficult decision the MKSC had to make when faced with very unwell patients. If these patients had any other solid tumour they would not be considered fit for chemotherapy, but in the setting of advanced HIV the poor PS could be ascribed to disseminated KS and they could obtain meaningful symptomatic improvement from even low doses of chemotherapy. No OS benefit was noted for the addition of RT to ART.

Other statistically significant associations on univariate analysis with inferior OS were S1 disease status (Fig. 1), any visceral involvement (Fig. 2), pulmonary involvement, PS $>2$, being on TB treatment and the presence of any OI. Most of these factors have been reported as known poor prognostic factors in AIDS-KS in the African setting. ${ }^{[5,6,16]}$ ACTG T1 and I1 disease was not associated with poor prognosis.

The lack of association between a CD4+ count $<150$ cells $/ \mu \mathrm{L}$ and survival along with the wide variation in $\mathrm{CD} 4+$ counts at diagnosis supports other reports that in African AIDS-KS the current ACTG cut-off of 150 cells/ $\mu \mathrm{L}$ for S1 disease may not be an appropriate prognostic marker. ${ }^{[11,19]}$ An alternative prognostic scale using incremental CD4+ counts, age, S1 disease and whether KS is the first AIDS-defining illness has been developed, but has not yet been validated in the African setting. ${ }^{[21]}$ It is notable that in the S1 subgroup, individual risk factors such as PS $>2$ and concurrent TB treatment were associated with poor survival. This association with poor survival was also true for visceral KS involvement, an entity that directly reflects disease burden but is not distinguished from other $\mathrm{T} 1$ presentations in the current ACTG staging system. These factors, particularly T1S1 disease stage and visceral KS, should be investigated to develop and validate prognostic indices for use in the sub-Saharan setting.

The issue of misdiagnosed pulmonary KS and PTB certainly warrants further study to determine the most cost-effective way of arriving at a diagnosis and thereby reducing delays in chemotherapy treatment, minimising unnecessary anti-TB treatment toxicity and improving prognostic accuracy.

\section{Study limitations}

The retrospective nature of the study lends itself to incomplete data collection and reporting bias as well as selection bias due to the site being a referral centre. Data on late relapse of KS, incidence of KS-IRIS and treatment toxicities were not known for all patients. Although verification of living status was reliably collected at time of censoring, the cause of death was not known in all patients. Owing to the small number of patients in the cohort, wide CIs were recorded during univariate analysis and it was found to be underpowered for multivariate analysis.

\section{Conclusion}

AIDS-KS affects young people in the Western Cape despite 10 years of ART roll-out. A significant proportion of patient are on concurrent TB treatment that could adversely affect diagnosis, adherence and response to treatment. A multidisciplinary approach to diagnosis, staging and treatment has been of benefit in this setting. Despite advanced disease at presentation and palliative treatment intent, survival outcomes are encouraging and seem to be positively affected by increased use of chemotherapy, despite suboptimal administration. Validation and exploration of prognostic indices specific to the subSaharan setting, particularly T1S1 disease status and visceral KS, would be valuable in designing appropriate treatment algorithms. Efforts should be made to improve adherence to oncological therapy and follow-up, and adequate provision should be made for long-term palliative supportive services for this patient group.

\section{Declaration. None.}

Acknowledgements. The MKSC acknowledges the continued dedication of all the referring clinicians in the subdistrict. The authors thank the Stellenbosch University Biostatistics Unit for assistance with statistical analysis of data.

Author contributions. The manuscript was prepared by HB and ZI and reviewed and edited by JJT.

Funding. Funding was received from the Division of Radiation Oncology, Department of Medical Imaging and Clinical Oncology, and the Division of Infectious Diseases, Department of Internal Medicine, Tygerberg Academic Hospital and Stellenbosch University.

Conflicts of interest. None.

1. Gbabe O, Okwundu C, Dedicoat M, Freeman E. Treatment of severe or progressive Kaposi's sarcoma in HIV-infected adults. Cochrane Database Syst Rev 2014, Issue 9. Art. No.: CD003256. https://doi. org/10.1002/14651858.cd003256.pub2

2. Chaabna K, Bray F, Wabinga H, et al. Kaposi sarcoma trends in Uganda and Zimbabwe: A sustained decline in incidence? Int J Cancer 2013;133(5):1197-1204. https://doi.org/10.1002/ijc.28125

3. Semeere A, Wenger M, Busakhala N, et al. A prospective ascertainment of cancer incidence in subSaharan Africa: The case of Kaposi sarcoma. Cancer Med 2016;5(5):914-928. https://doi.org/10.1002/ cam 4.618

4. Mosam A, Carrara H, Shaik F, et al. Increasing incidence of Kaposi's sarcoma in black South Africans in KwaZulu-Natal, South Africa (1983 - 2006). Int J STD AIDS 2009;20(8):553-556. https://doi. in KwaZulu-Natal, South Af

org/10.1258/ijsa.2008.008372

. Chu K, Mahlangeni G, Swannet S, Ford N, Boulle A, van Cutsem G. AIDS-associated Kaposi's sarcoma is linked to advanced disease and high mortality in a primary care HIV programme in South Africa. J Int AIDS Soc 2010;13(1):23. https://doi.org/10.1186/1758-2652-13-23

6. Sengayi M, Kielkowski D, Egger M, Dreosti L, Bohlius J. Survival of patients with Kaposi's sarcoma in the South African antiretroviral treatment era: A retrospective cohort study. S Afr Med J 2017;107(10):871-876. https://doi.org/10.7196/SAMJ.2017.v107i10.12362

7. Mosam A, Uldrick T, Shaik F, Carrara H, Aboobaker J, Coovadia H. An evaluation of the early effects of a combination antiretroviral therapy programme on the management of AIDS-associated Kaposi's sarcoma in KwaZulu-Natal, South Africa. Int J STD AIDS 2011;22(11):671-673. https://doi. org/10.1258/ijsa.2009.009145

8. Rohner E, Valeri F, Maskew M, et al. Incidence rate of Kaposi sarcoma in HIV-infected patients on antiretroviral therapy in southern Africa: A prospective multi-cohort study. J Acquir Immune Defic antiretroviral therapy in southern Africa: A prospective multi-cohort study. J
Syndr 2014;67(5):547-554. https://doi.org/10.1097/qai.0000000000000360

9. Krown S, Testa M, Huang J. AIDS-related Kaposi's sarcoma: Prospective validation of the AIDS Clinical 9. Krown S, Testa M, Huang J. AIDS-related Kaposi's sarcoma: Prospective validation of the AIDS Clinical
Trials Group staging classification. J Clin Oncol 1997;15(9):3085-3092. https://doi.org/10.1200/ jco.1997.15.9.3085

10. Fenner L, Reid S, Fox M, et al. Tuberculosis and the risk of opportunistic infections and cancers in HIV-infected patients starting ART in southern Africa. Trop Med Int Health 2013;18(2):194-198. https://doi.org/10.1111/tmi.12026

11. Mosam A, Hurkchand H, Cassol E, et al. Characteristics of HIV-1-associated Kaposi's sarcoma among women and men in South Africa. Int J STD AIDS 2008;19(6):400-405. https://doi.org/10.1258/ ijsa.2008.007301

12. Bohlius J. Kaposi's sarcoma in HIV-infected patients in South Africa: Multi-cohort study in the antiretroviral therapy era. Int J Cancer 2014;135(11):2644-2652. https://doi.org/10.1002/ijc.28894

13. Statistics South Africa. Census 2011: Census in brief. Pretoria: Stats SA, 2012. http://www.statssa.gov $\mathrm{za} /$ ?page $\_$id $=3955$ (accessed 26 October 2018). 
14. Unemori P, Leslie K, Hunt PW, et al. Immunosenescence is associated with presence of Kaposi's sarcoma in antiretroviral treated HIV infection. AIDS 2013;27(11):1735-1742. https://doi.org/10.1097/ qad.0b013e3283601144

15. Nasti G. AIDS-related Kaposi’s sarcoma: Evaluation of potential new prognostic factors and assessment of the ACTG staging system in the Haart era - the Italian Cooperative Group on AIDS and Tumors and the Italian Cohort of Patients Naïve From Antiretrovirals. J Clin Oncol 2003;21(15):2876-2882. https:// doi.org/10.1200/jco.2003.10.162

16. Sissolak G, Mayaud P. AIDS-related Kaposi's sarcoma: Epidemiological, diagnostic, treatment and control aspects in sub-Saharan Africa. Trop Med Int Health 2005;10(10):981-992. https://doi. org/10.1111/j.1365-3156.2005.01491.x

17. Bower M. British HIV Association guidelines for HIV-associated malignancies 2014. HIV Med 2014;15(Suppl 2):1-92. https:///doi.org/10.1111/hiv.12136

18. Bower M, Weir J, Francis N, et al. The effect of HAART in 254 consecutive patients with AIDS-related Kaposi's sarcoma. AIDS 2009;23(13):1701-1706. https://doi.org/10.1097/qad.0b013e32832d080d
19. Mosam A. A randomized controlled trial of highly active antiretroviral therapy versus highly active antiretroviral therapy and chemotherapy in therapy-naive patients with HIV-associated Kaposi antiretroviral therapy and chemotherapy in therapy-naive patients with HIV-associated Kaposi
sarcoma in South Africa. J Acquir Immune Defic Syndr 2012;60(2):150-157. https://doi.org/10.1097/ qai.0b013e318251aedd

20. Agaba P, Sule H, Ojoh R, et al. Presentation and survival of patients with AIDS-related Kaposis Agaba P, Sule H, Ojoh R, et al. Presentation and survival of patients with AIDS-related Kaposis
sarcoma in Jos, Nigeria. Int J STD AIDS 2009;20(6):410-413. https://doi.org/10.1258/ijsa.2008.008353 sarcoma in Jos, Nigeria. Int J STD AIDS 2009;20(6):410-413. https://doi.org/10.1258/ijsa.2008.008353
Stebbing J. A prognostic index for AIDS-associated Kaposi's sarcoma in the era of highly Stebbing J. A prognostic index for AIDS-associated Kaposis sarcoma in the era of highly
active antiretroviral therapy. Lancet 2006;367(9521):1495-1502. https://doi.org/10.1016/01406736(06)68649-2

Accepted 28 May 2018 